\title{
"Management Of Ankyloglossia" - Case Reports
}

\author{
Dr. Verdine Virginia Antony ，Dr. Rahamathulla Khan \\ 1 ( Department of Periodontics, Sirte University, Libya.) \\ 2 ( Department of Orthodontics, Sirte University, Libya.)
}

\begin{abstract}
Tongue tie or ankyloglossia is a developmental anomaly of the tongue characterized by an abnormally short, thick lingual frenum resulting in limitation of tongue movement. It can be categorized into 2 types- Total ankyloglossia and Partial ankyloglossia. Ankyloglossia can affect feeding, speech, and oral hygiene as well as have mechanical/social effects.

This article reports two cases of ankyloglossia which was surgically treated with uneventful healing and good patient satisfaction.
\end{abstract}

Key words:, Ankyloglossia, frenum, speech, surgical, tongue tie.

\section{Introduction}

The tongue is an important oral structure that is responsible for speech, swallowing and positioning of the teeth. Tongue tie or ankyloglossia is a developmental anomaly of the tongue characterized by an abnormally short, thick lingual frenum resulting in limitation of tongue movement. Etymologically ankyloglossia comes from a Greek word agkylos-"crooked", glossa-"tongue". It can be categorized into 2 types. Total ankyloglossia is rare and occurs when the tongue is completely fused to the floor of the mouth. Partial ankyloglossia is variable and encompasses the remainder of the cases [1].

The incidence of tongue tie varies from $0.2 \%$ to $5 \%$. Studies have shown a significant predilection for male child [2]. This may also occur with increased frequency in various syndromes including Smith-LemliOpitz syndrome,[3] Orofacial digital syndrome, Beckwith Weidman syndrome, Simpson-Golabi-Behmel syndrome[4] and X linked cleft palate.[5] Ankyloglossia can affect feeding, speech, oral hygiene as well as have mechanical/social effects When the tongue is retruded it causes blanching of the soft tissue and also exerts excessive force on the mandibular anteriors. The lingual frenum may cause midline diastema in lower central incisors. Ankyloglossia can also prevent the tongue from contacting the anterior palate, leading to a retained infantile swallow, resulting in an open bite deformity[6].

This paper reports surgical management of ankyloglossia in two young male patiens who had severe restriction of their tongue movements.

\section{CASE 1:}

\section{Case Reports}

A 22 year old male patient reported to the Department of Periodontics, with complaint of difficulty in complete protrusion of the tongue. Medical history was noncontributory. On intraoral examination the patient was diagnosed with class II ankyloglossia [Figure: 1] by utilizing Kotlow assessment [Table: 1]. There was no recession in relation to mandibular incisors lingualy. Surgical frenectomy of the lingual frenum was planned. The patient was informed about the treatment procedure and informed consent was obtained.

\section{CASE 2:}

A young male patient aged 16 years reported with a difficulty with difficulty in speech. Medical history was noncontributory. On intraoral examination the patient was diagnosed with class III ankyloglossia [Figure: 2] by utilizing Kotlow assessment [Table: 1]. There was no recession in relation to mandibular incisors lingualy. When the patient was asked to retrude the tongue, slight blanching was seen lingual to the anterior teeth with midline diastema in lower anteriors. Surgical frenectomy of the lingual frenum was planned. The patient was informed about the treatment procedure and informed consent was obtained.

\section{Treatment}

The surgical procedures for both the above mentioned cases where carried out in the same manner. The lingual frenectomy was undertaken under local anesthesia with $2 \%$ lignocaine and 1: 80000 adrenaline. After anesthesia was found to be effective, a suture was used at the tip of the tongue to stabilize it. [Figure: 3, 4] As the frenum became prominent, a No 15 blade was used to make incisions on the either side of the frenum .A diamond shaped wound was made and the frenum was removed [Figure 5,6]. The blunt end of the instrument was used to relieve the pull by muscle fibres so that tension free closure of the wound edges can be obtained. 
The wound was approximated with 3-0 black braided silk sutures

[Figure: 7, 8]. the favorable outcome of the procedure was apparent immediately and the extent of release could be assessed during the intervention itself . Postsurgical instructions were given along with a course of non-steroidal anti-inflammatory drugs for three days. The sutures were removed one week following the procedure. The post-operative period was uneventful with good healing and no scar formation.

The following exercises were advised: 1) Stretch the tongue up towards the nose, then down towards the chin and repeat, 2) Open the mouth widely and touch the big front teeth with the tongue with mouth still open, 3) Close the mouth and poke the tongue into the left and right cheek to make a lump: for 3 to 5 minute bursts, once or twice daily for 3 or 4 weeks post-operatively. The routine follow up at 4 weeks showed an extremely happy patient with improved tongue protrusion [Figure: 9, 10] and normal speech.

\section{Discussion}

Ankyloglossia is a rare congenital oral anomaly that causes difficulty in breast feeding and speech articulation. This anomaly is characterized by the attachment of the tongue to the floor of the mouth. The condition is the result of a failure in cellular degeneration leading to a much longer anchor between the floor of the mouth and the tongue [3]. Ankyloglossia represents a typical interdisciplinary problem concerning different specialties in dentistry. In many individuals ankyloglossia is asymptomatic and may resolve spontaneously. Therefore the surgery for ankyloglossia should be considered at any age depending on patient's history of speech, mechanical and social difficulty.

Surgical techniques for the therapy of tongue-ties can be classified into three procedures. Frenotomy is a simple cutting of the frenulum (of neonates). Frenectomy is defined as complete excision, i.e., removal of the whole frenulum (at or after 6 months of age). Frenuloplasty involves various methods to release the tongue-tie and correct the anatomic situation [7]. In addition to surgical intervention, LASER and electrocautery are also being used for treating ankyloglossia[8]. The cases presented in this paper were treated with transverse-vertical release frenectomy which resulted in good treatment outcome and patient satisfaction with improved tongue movements and correction of speech problems.

Post-operative exercise following tongue-tie surgery were not intended to increase muscle-strength, but to: i) Develop new muscle movements, particularly those involving tongue-tip elevation and protrusion, inside and outside of the mouth, ii) Increase kinesthetic awareness of the full range of movements the tongue and lips can perform, iii) Encourage tongue movements related to cleaning the oral cavity, including sweeping the insides of the cheeks, fronts and backs of the teeth, and licking right around both lips[6,8].

\section{TABLE}

TABLE: 1 Kotlow's Classification based on free tongue

\begin{tabular}{|l|l|l|}
\hline \multicolumn{2}{|c|}{ CLASSIFICATION OF ANKYLOGLOSSIA } & Range of free tongue* \\
\hline \multicolumn{2}{|c|}{ Normal } & $>16 \mathrm{~mm}$ \\
\hline Class I & Mild ankyloglossia & $12-16 \mathrm{~mm}$ \\
\hline Class II & Moderate ankyloglossia & $8-11 \mathrm{~mm}$ \\
\hline Class III & Severe ankyloglossia & $3-7 \mathrm{~mm}$ \\
\hline Class IV & Complete ankyloglossia & $<3 \mathrm{~mm}$ \\
\hline
\end{tabular}

*Free-tongue is measured from the insertion of the lingual frenum into the base of the tongue to the tip of the tongue

\section{Conclusion}

If severe/complete ankyloglossia is present in adults there is usually an obvious limitation of the tongue protrusion, elevation and speech problems. Optimal management of tongue tie includes timely and appropriate surgical intervention followed by speech therapy if required, ultimately delivering pleasing results, in a shorter time than expected.

\section{References}

[1]. Lawrence A. Kotlow Ankyloglossia (tongue-tie): A diagnostic and treatment quandary. Quintessence International 1999; 30:259-262

[2]. Tanaj, chaubal, maladixit Ankyloglossia and its management. J Indian Soc Periodontal 2011: vol 15;270-272

[3]. Fisher SE, Frame JW Electrosurgical management of soft tissues and restorative dentistry. Dent clin North Am vol 24:247; 1980

[4]. Suter VGA and Bornstein MM. Ankyloglossia: Facts and Myths in Diagnosis and Treatment. J Periodontol 2009;80:1204-1219.

[5]. Lalakea, M. Lauren, Messner, Anna H. Ankyloglossia: The adolescent and adult perspective. Otolaryngology - Head and Neck Surgery. 2003;128:746-752

[6]. Suter VG, Bornstein MM Ankyloglossia: facts and myths in diagnosis and treatment J Periodontol. 2009 Aug;80(8):1204-19

[7]. Messner AH, Lalakea ML. Ankyloglossia: controversies in management. International Journal of Pediatric Otorhinolaryngology 2000; 54(2-3):123

[8]. Tuli A, Singh A. Monopolar diathermy used for correction of ankyloglossia. Journal of Indian Society of Pedodontics and Preventive Dentistry 2010; 28(2):130. 


\section{LEGENDS FOR PICTURES:}

CASE: 1

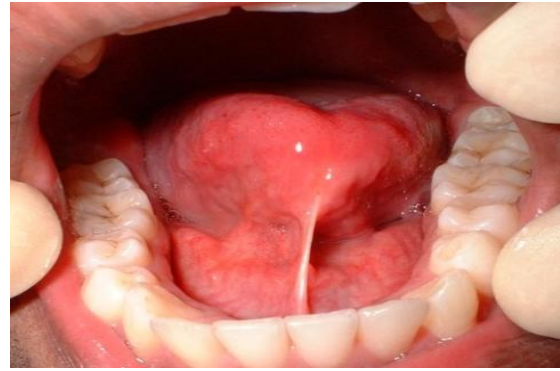

Figure 1:

Pre-operative -Showing Class II ankyloglossia

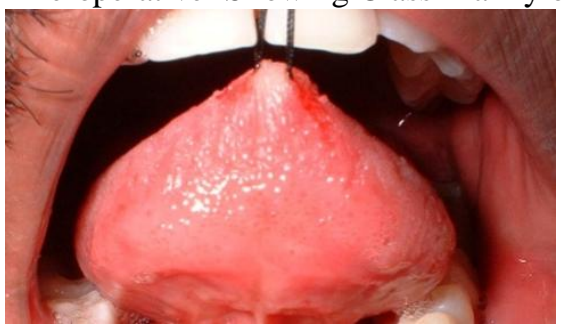

Figure 3:

Tongue stabilized in position

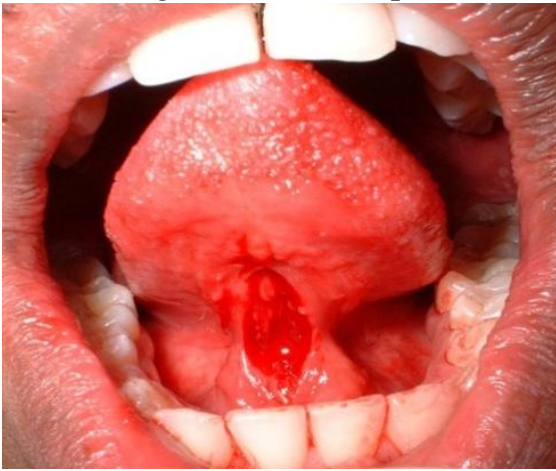

Figure 5:

Excision of frenum

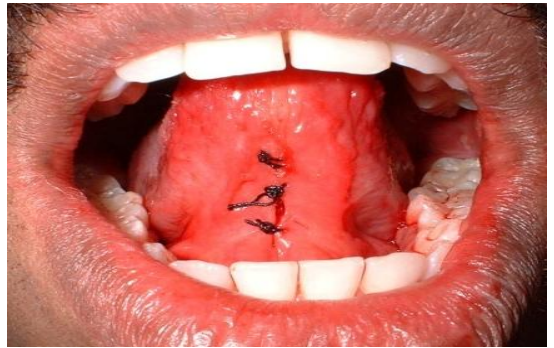

Figure 4:

Margins of the incision sutured

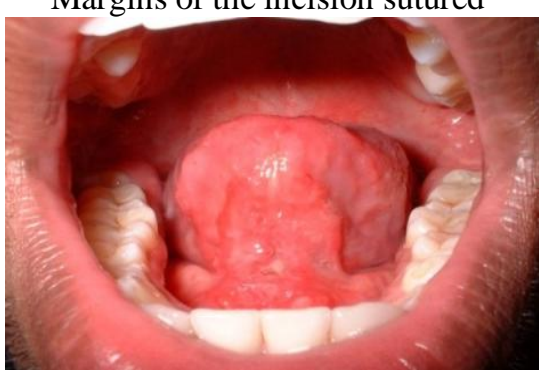

Figure 9:

4 Weeks post-operative view
CASE: 2

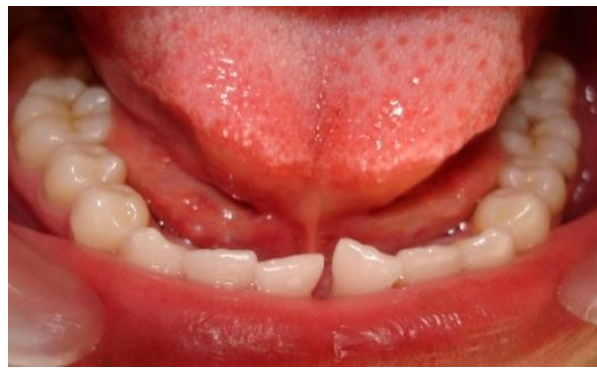

Figure 2:

Pre-operative- Showing Class III ankyloglossia

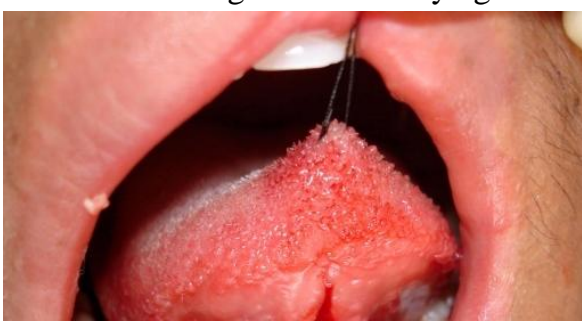

Figure 4:

Tongue stabilized in position

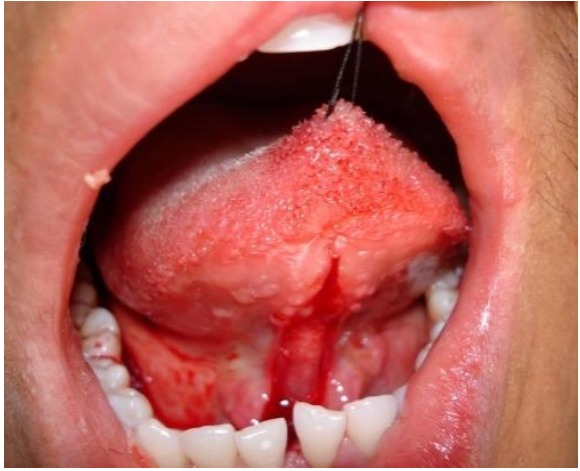

Figure 6:

Excision of frenum

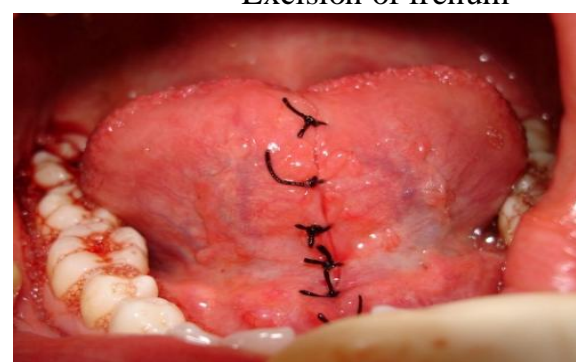

Figure 4:

Margins of the incision sutured

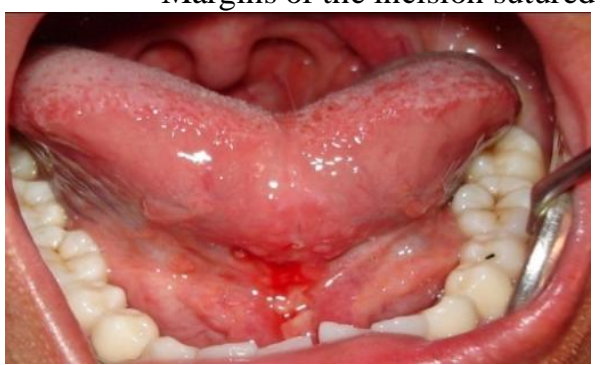

Figure 10:

4 Weeks post-operative view 